\title{
SENSIBILIZAÇÃO DE AGENTES COMUNITÁRIAS DE SAÚDE SOBRE CAPTAÇÃO PRECOCE DE GESTANTES
}

\author{
AWARENESS OF COMMUNITY HEALTH STAFF ON FUNDING OF EARLY \\ PREGNANCY \\ Bruno Ronchi de Souza ${ }^{1}$ \\ Brunella Flores Pupo ${ }^{2}$ \\ Eveline Geller ${ }^{2}$ \\ Francielle Lazzarin de Freitas Gava ${ }^{2}$ \\ Ioná Vieira Bez Birolo
}

\begin{abstract}
RESUMO
Trata-se de um estudo exploratório, qualitativo, resultado de um projeto de extensão da Universidade do Extremo Sul Catarinense - UNESC, UNASAU, curso de enfermagem, com objetivo de identificar de que forma as Agentes Comunitárias de Saúde (ACS) de uma Estratégia Saúde da Família (ESF) de um município do extremo sul catarinense fazem a captação precoce de suas gestantes a partir de seis questões aplicadas durante um encontro para sensibilização das mesmas. Participaram 33 ACS. Os dados qualitativos foram categorizados por análise de conteúdo de Minayo. Observou-se que as ACS estão bem informadas sobre sua atuação nas unidades de saúde, porém não conheciam muito sobre a Rede Cegonha. Assim evidencia-se a necessidade de realizar mais oficinas de discussão para aprimorar o aprendizado e instrumentar esses profissionais tão importantes para o vínculo da comunidade com as unidades de saúde.

Palavras-chave: Rede Cegonha; ACS; Gestantes.
\end{abstract}

\begin{abstract}
This is an exploratory study, qualitative result of an extension project of the University of Southern Santa Catarina-UNESC Far, UNASAU- nursing course in order to identify how the agents Community Health (CHA) a Family Health Strategy (FHS) a city in the extreme south of Santa Catarina make early identification of pregnant women from their six questions applied during a meeting to raise awareness of the same. 33 attended ACS. Qualitative data were categorized by content analysis Minayo. It was observed that the ACS are well informed about their activities in health facilities, but did not know much about the Stork Network. Thus highlights the need for more workshops to enhance discussion and learning this instrument as important for the professional bond between the community and health facilities Keywords: Stork Network; ACS; Pregnant.
\end{abstract}

\footnotetext{
1 Autor para correspondência: Universidade do Extremo Sul Catarinense - Criciúma Endereço: Rua Martin Afonso de Souza, 535, Operária Nova, Criciúma, SC. E-mail: brunoronchi_2000@hotmail.com.

${ }^{2}$ Universidade do Extremo Sul Catarinense - Criciúma.
} 


\section{INTRODUÇÃO}

O Brasil possui como sistema operacional para a saúde pública o Sistema Único de Saúde (SUS), o qual foi estabelecido conforme a Lei Orgânica N. ${ }^{\circ}$ 8.080, de 19 de setembro de 1990, possuindo como princípios e diretrizes a universalidade, igualdade, integralidade, hierarquização, regionalização, resolutividade, descentralização, complementaridade do setor privado e participação dos cidadãos (BRASIL, 1990).

A situação atual da saúde no nosso país é a de aumento das condições crônicas pelas alterações demográficas, na alternância dos padrões de consumo e nos estilos de vida, junto com a urbanização acelerada e as estratégias mercadológicas (MENDES, 2012).

Para a Organização Pan-Americana da Saúde (2011) a estratégia das redes de atenção coordenadas pela Atenção Primária à Saúde (APS) é o princípio essencial para enfrentar o complexo cenário contemporâneo das necessidades de saúde e acabar com a fragmentação que enfraquece a maioria dos sistemas de saúde do mundo, incluindo o Brasil.

Segundo o Ministério da Saúde (2010), existem atributos das Redes de Atenção a Saúde, que são a população; além de territórios bem definidos proporcionadores da determinação da oferta de serviços de saúde; APS sendo o primeiro nível de atenção e entrada do sistema; sendo formada por múltiplos trabalhadores da área da saúde, capacitados a atender toda a população; vários estabelecimentos de saúde; mecanismos de coordenação; cuidado continuado e integração da assistência; também possuir local adequado para atendimento especializado; atenção à saúde com foco no indivíduo, família e comunidade; definição de metas e avaliação dos gerentes com uma grande participação social; a rede deve possuir uma governança única com uma missão; articulação de políticas institucionais; gestão integrada dos sistemas de apoio e administrativo, clínico e logístico; suficiência nos recursos humanos; financiamento tripartite; sistema de informação integrado; gestão baseada em resultados e ação intersetorial. Com as RASs possuindo o objetivo de garantir a integridade do cuidado.

Já a Organização Mundial da Saúde afirma que as Redes de Atenção à saúde possuem seis modelos integrativos: intervenções preventivas e curativas para certa população; espaços de integração de vários serviços; a continuidade de atenção à saúde; trabalho intersetorial; vinculação entre política de saúde e a gestão; integração vertical nos níveis de atenção (WORLD HEALTH ORGANIZATION, 2008b).

A constituição das RASs é essencialmente feita por três setores: a população, a estrutura operacional que se distribui por: atenção primária à Saúde, os sistemas de apoio, os 
sistemas logísticos, o sistema de transporte em saúde, e o sistema de governança das redes; e, finalmente, os modelos de atenção à saúde aguda e crônica (MENDES, 2012. p. 20).

A Rede cegonha, regida pelo governo Federal a partir da Portaria 1459 de 24 de junho de 2011, possui como objetivos aperfeiçoar o Sistema Único de Saúde (SUS) em todo o território Nacional (SECRETARIA DO ESTADO DE SAÚDE DE SANTA CATARINA, 2012).

Além disso, objetiva: Reduzir a mortalidade materna e infantil com ênfase no componente neonatal; fomentar a implementação de novo modelo de atenção à saúde da mulher e à saúde da criança com foco na atenção ao parto, ao nascimento, ao crescimento e ao desenvolvimento da criança de zero aos vinte e quatro meses; e organizar a Rede de Atenção à Saúde Materna e Infantil para que esta garanta acesso, acolhimento e resolutividade (BRASIL, 2011).

Tem como composição medidas que visam garantir atendimento adequado a todas as brasileiras, além de atendimento seguro e humanizado desde que a gestante descobre a gravidez até pré-natal e parto além de garantir a saúde até os dois primeiros anos de vida da criança (BRASIL, 2011).

O Brasil conseguirá atingir o quarto Objetivo de Milênio (ODM) até 2015, o qual visa reduzir em dois terços a taxa de mortalidade na infância (BRASIL, 2009).

Assim existem quatro componentes, os quais compreendem uma série de ações de atenção à saúde nessa estratégia: Pré-natal; Parto e nascimento; Puerpério e Atenção Integral à Saúde da Criança e Sistema Logístico, Transporte Sanitário e Regulação (BRASIL, 2011).

Essa Rede visa vincular a gestante à unidade de referência para que a assistência tenha qualidade, e garantir transporte seguro e implementar a atenção ao parto e nascimento com qualidade, além de direito a acompanhante no momento do parto. A Rede Cegonha possuirá financiamento pelo Ministério da Saúde, com aporte ao pré-natal, transporte, centro de parto normal e casa da gestante, bebê e puérpera e leitos (BRASIL, 2011).

Para sua operacionalização, foram elaboradas cinco fases, as quais devem ser seguidas pelos Municípios que irão aderir à Rede Cegonha, que são: Adesão e diagnóstico; Desenho Regional da Rede Cegonha; Contratualização dos Pontos de Atenção; Qualificação dos componentes; e Certificação (BRASIL, 2011).

A Matriz Diagnóstica será feita por quatro componentes, os quais também servirão para a priorização epidemiológica da Rede Cegonha: Indicadores de morbi e mortalidade; Indicadores de atenção; Situação da capacidade instalada hospitalar; e Indicadores de gestão (BRASIL, 2011). 
Existem trabalhos exitosos na humanização de atenção ao parto e nascimento, como o Hospital Sofia Feldman (Belo Horizonte - Minas Gerais) e São Pio X (Ceres - Goiás) (RATTNER, 2011).

Assim a Rede Cegonha será implementada gradativamente, com início nas regiões que possuem maior taxa de morbidade e mortalidade materna e infantil, e em concordância com critérios epidemiológicos e de densidade populacional para gerir prioridades da atuação da Rede, com a possibilidade de todos os Municípios aderirem à Rede (SECRETARIA DO ESTADO DE SAÚDE DE SANTA CATARINA, 2012).

A Rede Cegonha, iniciativa estabelecida pelo Sistema Único de Saúde (SUS) com a Portaria 1459 de 24 de junho de 2011 (BRASIL, 2011), objetiva garantir a todas as brasileiras: atendimento adequado, seguro e humanizado desde a confirmação da gravidez, passando pelo pré-natal e o parto, até os dois primeiros anos de vida do bebê. Também visa garantir à gestante acolhimento com classificação de risco, vinculação a unidade de referência com transporte seguro, manutenção de direitos sexuais e reprodutivos das brasileiras, além de reduzir taxas de mortalidade materna e neonatal (SECRETARIA DO ESTADO DE SAÚDE DE SANTA CATARINA, 2012).

A gestação compreende momentos de transformações corporais, psíquicas, sociais e familiares (FALCONE et al., 2005). De acordo com Brasil (2006) é fundamental que seja disponibilizada à gestante e à família uma atenção pré-natal e puerperal humanizada e de qualidade. A assistência neste caso deve ser construída na compreensão da integralidade corpo e mente, na percepção da influência ambiental, física, social, econômica e cultural das diversas pessoas envolvidas.

Sabe-se que $92 \%$ das mortes associadas ao período gravídico-puerperal e aborto são evitáveis (BRASIL, 2006). E, neste sentido, a assistência durante o período pré-natal pode privilegiar se bem conduzida a discussão de questões únicas para a mulher, o parceiro e a família (VEZOZZO; KIMINAMIE; RADIGONDA, 2008).

A morte materna e neonatal pode ser considerada um dos maiores desafios em relação ao período aqui discutido. Em 2003 a Razão de Morte Materna (RMM) foi de 51,74 óbitos. Outro dado relevante diz respeito à morte de crianças menores de cinco anos de idade no período de um ano. Estimava-se que 52\% destas ocorriam no período neonatal e que grande parte delas estava associada à atenção dispensada à gestação, ao parto e ao puerpério (BRASIL, 2006).

Diante desses fatos, fica demonstrada a necessidade de empenho coletivo, de setores governamentais e não governamentais, para a melhoria e qualificação da atenção pré-natal e 
puerperal em todo o País. Estas podem incluir melhorias nas ações de promoção e prevenção de agravos à saúde, além de acesso ao diagnóstico e tratamentos apropriados às intercorrências que possam ocorrer neste período (BRASIL, 2006).

Durante a atenção obstétrica e neonatal, características como a qualidade e a humanização dos serviços são essenciais. A mulher, o recém-nascido e a família devem ter acesso a serviços e profissionais que os acolham com dignidade e enfoque-os como sujeitos de direitos. Para tal, considera o outro como sujeito e não como objeto passivo. E que, assim, possam ser construídas redes de atenção que sustentem o processo com humanização e qualidade da assistência prestada (BRASIL, 2006).

Enfatiza-se que a necessidade de assistir com excelência a mulher, a criança e a família envolvida no ciclo gravídico puerperal está contida na estratégia denominada Rede Cegonha do Ministério da Saúde decretada pela portaria ministerial $N^{\circ} 1.459$, de 24 de junho de 2011 (BRASIL, 2011).

Em termos gerais, em relação à gestação, é recomendado pelo Ministério da Saúde brasileiro o início da atenção obstétrica à mulher no primeiro trimestre e, ao final da gestação, tenham sido realizadas no mínimo seis consultas pré-natais. São indicados também: exame clínico das mamas da gestante, exame colpocitopatológico do colo do útero, triagem e detecção precoce da Doença Hipertensiva Específica da Gestação, exame de tipagemsanguínea, hemograma, anti-HIV, sorologia para sífilis e toxoplasmose. Devem ser realizadas também a suplementação com ácido fólico e sulfato ferroso e a imunização da gestante para o tétano (GONÇALVES; CESAR; MENDONZA-SASSI, 2009; BRASIL, 2011).

São imprescindíveis neste momento o acolhimento com classificação de risco, garantia de exames pré-natais de alto risco e vinculação da UBS ao Centro obtétrico/maternidade onde será realizada a atenção no momento do parto (BRASIL, 2011).

Para a garantia de um parto seguro a portaria da rede Cegonha prevê como necessário a suficiência de leitos hospitalares, ambiência, direito a acompanhante, acolhimento com classificação de risco, boas práticas baseadas em segurança para o binômio mãe e filho e práticas de gestão baseadas no cuidado horizontal com a presença de conselho gestor (BRASIL, 2011).

Em relação ao puerpério e atenção a criança é fundamental que sejam estabelecidos o aleitamento materno, acompanhamento da criança no mínimo até os 24 meses, visita domiciliar na primeira semana, busca ativa de crianças vulneráveis ou em risco e planejamento reprodutivo nas comunidades (BRASIL, 2011). 
Entende-se que para a efetivação da proposta ainda são necessários maiores esforços e organização dos serviços, sempre almejando propiciar de maneira integral e efetiva a qualidade e humanização da assistência prestada.

\section{PROCEDIMENTOS METODOLÓGICOS}

A abordagem da pesquisa foi qualitativa, porque os objetivos foram identificar o grau de conhecimento que as Agentes Comunitárias de Saúde tinham antes de ser iniciada a sensibilização sobre a captação precoce de gestantes antes do terceiro trimestre, conforme preconizado pela Rede cegonha, para conhecer onde encontra-se a principal dificuldade de atuação dessas profissionais.

Segundo Leopardi (2002, p. 117), na pesquisa qualitativa, compreende-se um problema no contexto em que se vive. No que tange a abordagem qualitativa, optou-se por esse enfoque para que houvesse a valorização das falas dos participantes e assim fossem expostos conceitos, pensamentos e opiniões, enfatizando a experiência cotidiana no cenário vivenciado.

O estudo foi desenvolvido durante um dos encontros com as Agentes Comunitárias de Saúde de algumas ESFs do município de Criciúma, por meio das seguintes questões levantadas à elas antes do início das discussões: Existem mulheres com suspeita de gravidez na sua comunidade e dificuldade para realizar o exame?; Como você identifica uma gestante?; Qual a sua primeira atitude após ter identificado a gestante?; Como você reconhece uma gestante de risco?; Como você faz o acompanhamento dessas gestantes até o parto?;Como você dá continuidade ao acompanhamento após o puerpério?. Cada ACS foi identificada pela letra A1, A2, A3, A4, sucessivamente, garantindo desta forma o anonimato dos participantes.

Para a realização da pesquisa os sujeitos do estudo assinaram um termo de consentimento, sendo que este assegura o sigilo da identidade dos participantes. O termo segue as exigências formais contidas na resolução 466/12, do Conselho Nacional de Saúde.

A análise e interpretação dos dados qualitativos foram realizadas, no que se refere aos dados qualitativos, pela categorização dos dados, por meio da ordenação, classificação e análise final dos dados pesquisados, proposta por Minayo: ordenação dos dados; classificação dos Dados; análise final (MINAYO, 2012, p. 78).

\section{APRESENTAÇÃO E DISCUSSÃO DOS DADOS}

O presente estudo revelou que dentre as 33 entrevistas, praticamente todas responderam que não há dificuldades das mulheres grávidas de realizar exames, porém 04 
responderam que em sua comunidade algumas mulheres têm dificuldades de realizar exames e justificam dizendo que essas mulheres em algumas vezes não tem documentos, são usuárias de drogas ou não querem que a família saiba de sua gestação. Evidenciado nas seguintes falas:

ACS 15: Sim por falta de documentação da paciente, geralmente quando a mesma é usuária de drogas da população e dos membros da equipe. Buscando qualidade de vida.

ACS 33: Sim ,quando ocorre na adolescência e quando esta ainda não revelado aos familiares.

Quando questionadas sobre como elas identificam uma gestante, 07 responderam que é através de exames, 22 ACS responderam que identificam uma gestante nova durante as visitas domiciliares mensais ou quando a mesma vem procurar os serviços da unidade de saúde, conforme falas a seguir:

ACS 12: Nas visitas domiciliares já há o relato ou quando ela procura a unidade de saúde.

ACS 13: Através de visita domiciliar.

ACS 15: Quando a paciente procura o atendimento médico da unidade de saúde, ou nas visitas domiciliares quando a mesma pede informações sobre o assunto.

ACS 19: Às vezes elas falam, mas muitas vezes quando marcam a primeira consulta na unidade de saúde.

Quando questionadas em relação a qual atitude adotam ao identificar uma gestante, 28 ACS responderam que encaminham a gestante para a unidade de saúde e avisam a enfermeira gerente da unidade, que fica claro nas afirmações a seguir:

ACS 01: Orientar paciente ir a unidade para realizar o pré-natal.

ACS 05: Levar a informação até a enfermeira e já marcar a consulta pra ela começar o pré-natal.

ACS 12: Se ela está suspeitando a gestação, orienta para que ela procure a unidade e fale com a nossa enfermeira para realização do exame e a consulta do pré-natal seja marcada. Se ela já é gestante oriento para realização do pré-natal, vacinas, etc.

Porém outras ACS responderam que iriam cadastrá-la como gestante, realizar o cadastro da ficha A, e do SISPRENATAL, conforme falas a seguir:

ACS 10: Cadastrá-la como gestante. 
ACS 13: Orientação de Acrescentar ela na ficha A como gestante, faço acompanhamento de gestante e peço para ela ir no posto com o resultado positivo, fazer o cadastro de gestante com a enfermeira.

ACS 20: Encaminho ao cadastro de SISPRENATAL e aconselho a procurar o médico na unidade para uma consulta.

Ao serem questionadas em relação de que maneira elas reconhecem uma gestante de risco, as respostas foram: 27 ACS responderam que procuram identificar fatores de risco como idade da gestante, se acima de 40 anos ou adolescente, uso de medicações, drogas, cigarro, bebida alcoólicas, fatores nutricionais, se tem hipertensão, diabetes, obesidade, história de outros abortos, convívio familiar, que se fazem presentes nas falas a seguir.

ACS 01: Quando a gestante apresenta queixas, como pressão alta, sangramento, paciente de menor, etc..

ACS 05: Se ela estiver com pressão alta, se é menor de idade, com mais idade, com diabetes.

ACS 08: Certificar sua pressão, se o peso é normal, se está com diabetes, história de abortos anteriores.

ACS 12: Meio social que a gestante vive, fatores patológicos, idade, estado nutricional, fatores estes identificados mediante a visita.

ACS 23: Meio social que a gestante vive, fatores patológicos, idade, estado nutricional, fatores estes identificados mediante a visita.

Algumas ACS relataram outras questões como conhecer sua micro-área, a família da gestante, por observar sinais de perigo e por más condições familiares, vida social da gestante, porém nãos descreveram com detalhes para que se pudesse entender melhor suas falas, como podem ver a seguir:

ACS 16: Por conhecer minha micro-área e por conhecer a família.

ACS 26: Observando os sinais de perigo em geral.

Ao serem questionadas sobre como fazem o acompanhamento das gestantes até o parto, 01 ACS não respondeu a pergunta e 32 ACS responderam que fazem o acompanhamento mensal das gestantes através de visitas domiciliares, onde verificam se o pré-natal está sendo realizado corretamente, conforme frases a seguir: 
ACS 13: Nas visitas domiciliares, fazendo o acompanhamento de gestante ou conversando com familiares se a gestante trabalha.

ACS 15: Com visitas domiciliares mensalmente, porém quando necessitado são mais de 03 visitas ao mês, fazendo busca ativa para as consultas e os grupos existentes na unidade de saúde.

ACS 21: Com visitas domiciliares uma vez por mês e quando for de risco mais de uma vez por mês.

ACS 28: Fazemos a visita domiciliar, preenchendo o acompanhamento da gestante, perguntando se ela está bem, se fez as vacinas, os exames obrigatórios com sífilis, HIV entre outros.

Quando questionadas sobre como dão continuidade ao atendimento após o puerpério, 32 ACS responderam que fazem visitas domiciliares logo após o parto, dão orientações sobre amamentação, alimentação, vacinas, consultas pediátricas, como pode ser observado nas falas:

ACS 12: A visita já é realizada logo após o nascimento da criança, e fornecido orientações sobre teste do pezinho, vacina, higiene da criança, entre outros temas pertinentes e para a mãe todo o cuidado pós parto, sobre novas rotinas, amamentação e sua importância.

ACS 19: VD com a enfermeira acompanhando a criança e oriento quanto a amamentação, vacinas e levar a criança para a primeira consulta ao pediatra e ela também na primeira consulta com médico.

ACS 28: Fazendo visitas domiciliares, orientando a puérpera a amamentar, orientando a levar a criança para fazer as vacinas, ir na consulta com o pediatra regularmente pelo menos uma vez por mês até os 12 meses, pesar a criança todo mês.

ACS 29: Continuo acompanhando a puérpera e o bebê, é realizado uma visita com a enfermeira e a ACS para avaliar o estado da puérpera e seu $R N$ e onde são reforçadas assuntos sobre amamentação há 01 ano.

Uma ACS relata outra situação, como podem perceber nesta fala: 
ACS 07: Só levo a enfermeira até a casa da puérpera pra visita, depois não faço mais nada.

As ACS até tem consciência que é necessário realizar o acompanhamento das crianças, porém não sabiam que é preconizado realizá-lo até a criança completar os dois anos de idade. Após o nascimento da criança, a unidade de saúde deve orientar a família sobre a amamentação e a introdução da alimentação complementar no período correto para contribuir para a formação de hábitos alimentares saudáveis desde a infância.

\section{CONCLUSÕES}

O estudo demonstrou que as ACS têm papel fundamental na captação das gestantes. A atuação das mesmas junto à equipe de saúde cria maior vínculo entre gestante e serviço de saúde, podendo assim efetivar um atendimento com qualidade e eficiência à mulher no seu período gravídico, puerperal e à criança.

As ACS mostraram conhecimento na captação das gestantes, porém durante as discussões foi visto o desconhecimento da Rede Cegonha. Isso pode estar relacionado à recente implantação das Redes, sendo necessária uma maior discussão tanto das Redes de Atenção à Saúde, como prioritariamente a Rede Cegonha com todos profissionais da área da saúde, a fim de conhecerem a organização e atuação das Redes.

Diante dos dados encontrados, pode-se perceber a peculiaridade como cada ACS atua dentro da sua realidade, porém ao mesmo tempo discutir de forma geral que artifícios podem ser usados e padronizados para que ocorra a captação precoce de gestantes. Surge então a proposta de novas discussões, que envolvam mais profissionais da atenção básica para que se torne eficiente a captação precoce das gestantes.

Observa-se a necessidade de educação continuada, para os profissionais que estão diretamente ligados à captação de gestantes, a puérpera, ao RN e à criança, para que possam melhorar suas ações, sabendo agir de forma a minimizar morbidades e a mortalidade, proporcionando uma melhor qualidade de vida.

Contudo durante a aplicação das propostas do projeto, pôde-se conhecer a realidade das ACS atuantes na captação das gestantes em cada região e as diferentes situações que existem em um município. Obteve-se exitosa experiência em trabalhar com as ACS, discutindo temas relacionados à Rede Cegonha, e reconhecer a necessidade de continuarem as discussões tanto com as ACS, como com demais profissionais da atenção básica a saúde. 


\section{REFERÊNCIAS}

BRASIL. Ministério da Saúde. Secretaria de Vigilância em Saúde. Departamento de Análise de Situação de Saúde. Saúde Brasil 2009: uma análise da situação de saúde e da agenda nacional e internacional de prioridades em saúde. Brasília (DF): 2010b. Disponível em:

<http://portal.saude.gov.br/portal/arquivos/pdf/29_11_10_saude_brasil_web.pdf>. Acesso em: 25 jan. 2013.

BRASIL. Portaria 1459 de 24 de junho de 2011 institui no âmbito do Sistema Único de Saúde, a Rede Cegonha. Disponível em:

<http://www.saude.pi.gov.br/documentos/2012/arquivos/rede_cegonha/portaria_Rede _Cegonha_1459.pdf>. Acesso em: 24 jan. 2013.

BRASIL. Ministério da Saúde. Secretaria de Atenção à Saúde. Departamento de Ações Programáticas Estratégicas. Área Técnica de Saúde da Mulher. Pré-natal e Puerpério: atenção qualificada e humanizada - manual técnico. Brasília: Ministério da Saúde, 2006.

BRASIL. Ministério da Saúde. Secretaria de Estado da Saúde. Proposta de formulação da Rede cegonha para a região metropolitana de Florianópolis. Florianópolis (SC): 2012.

BRASIL. Lei n 8080 de 19 de setembro de 1990. Dispõe sobre as condições para a promoção, proteção e recuperação da saúde, a organização e o funcionamento dos serviços correspondentes e dá outras providências. Brasília (DF): Guia do Conselheiro, 2002.

FALCONE, Vandra Mafra; MÄDER, Custódia Virginia de Nóbrega; LIMA, Christianne Freitas; NASCIMENTO, Joacira Mota Matos Santos; NÓBREGA, Fernando José de. Atuação multiprofissional e a saúde mental de Gestantes. Revista Saúde Pública, v. 39, n. 4, p. 612-618, 2005.

GONÇALVES, Carla Vitola; CESAR, Juraci Almeida; MENDONZA-SASSI, Raul A. Qualidade e equidade na assistência a gestante: um estudo de base populacional no Sul do Brasil. Rio de Janeiro. Cad. De Saúde Publica, v. 25, n. 11, p. 2507-2516, Nov. 2009.

MENDES, E. V. As Redes de Atenção a Saúde. 2. Ed. Brasília-DF: Organização PanAmericana da Saúde 2011, p.28-29.

ORGANIZAÇÃO MUNDIAL DA SAÚDE. Cuidados inovadores para condições crônicas: componentes estruturais de ação. Brasília, Relatório Mundial. Brasília Organização Mundial da Saúde, 2003.

RATTNER, D. Humanização na atenção a nascimentos e partos: ponderações sobre políticas públicas. 2009. Disponível em:<http://dx.doi.org/10.1590/S141432832009000500027>. Acesso em: 03 jan. 2013.

VEZOZZO, Kátia Mara Kreling; KIMINAMI, Nathalia; RADIGONDA, Heloise Pinhas. Atendimento de Enfermagem a Gestantes na Unidade Básica de Saúde (UBS). II Encontro de Extensão da UniFil - 10 e 11 de Novembro de 2008. Disponível em: <http://web.unifil.br/docs/extensao/II/04_Atendimento\%20de\%20enfermagem_a_gest ante_na_UBS.pdf $>$. Acesso em: $20 \mathrm{fev} .2012$. 
WORLD HEALTH ORGANIZATION Integrated health services: what and why? Geneva, World Health Organization, Technical Brief $n^{\circ}$ 1, 2008b.

LEOPARDI, Maria Tereza. Metodologia da Pesquisa na Saúde. Florianópolis: UFSC, 2002.

MINAYO, Maria Cecilia de Souza. Pesquisa social: teoria, método e criatividade. 28 ed. Petrópolis, RJ: Vozes, 2009.

MINAYO, Maria Cecilia de Souza; SANCHES, Odécio. Quantitativo-Qualitativo: Oposição ou Complementaridade. Caderno de Saúde Pública, Rio de Janeiro, v. 9, n. 3, p. 239262, jul/set 1993. 